\title{
The ionosphere electron density spatio-temporal modeling based on the Slepian basis functions
}

\author{
Mohammad Ali Sharifi ${ }^{1,2} \cdot$ Saeed Farzaneh $^{1}$
}

Received: 5 March 2015/Accepted: 14 February 2016/Published online: 25 February 2016

(C) Akadémiai Kiadó 2016

\begin{abstract}
Radio occultation (RO) is one of the most novel satellite approaches that can be used to study ionospheric electron densities, by combining the observations of global navigation satellite systems and low earth orbiting (LEO) satellites to determine ionospheric electron density profiles with high vertical resolutions. This study aims at developing a multi-dimensional model of the electron density derived from ionospheric GPS RO measurements by the FORMOSAT-3/COSMIC satellites. In order to develop a 4D local model for the electron density, a Slepian function was used to account for longitudinal and latitudinal variations, the B-spline was utilized for time variations and the Chapman profile function was used to express the height variations of the electron density, in this way the parameters of the Chapman function, namely the maximum electron density of the F2 layer $(N m F 2)$ and the corresponding F2 peak height $(h m F 2)$ were modeled. The coefficients of the two sets of the Slepian functions were estimated using the least-squares adjustment technique. In order to validate the accuracy of the proposed technique, two approaches are considered: (1) The estimated F2-peak values were compared with the IRI model and ionosonde stations values, (2) Comparing the modeled electron density profile against the one estimated using RO methods.
\end{abstract}

Keywords Chapman function - Ionosphere electron density - GPS radio occultation · Slepian function - FORMOSAT-3/COSMIC

Saeed Farzaneh

farzaneh@ut.ac.ir

Mohammad Ali Sharifi

sharifi@ut.ac.ir

1 School of Surveying and Geospatial Engineering, College of Engineering, University of Tehran, Tehran, Iran

2 Research Institute of Geoinformation Technology (RIGT), College of Engineering, University of Tehran, Tehran, Iran 


\section{Introduction}

The ionosphere, which is a layer of the ionised gas in the upper layers of the atmosphere around the Earth, plays a critical role in satellite communication, military communications and space science. The influence that the ionosphere has on communications systems can be quantified, provided that the distribution of the electron density within the ionosphere is known. Several methods and instruments exist, which can be used to determine the distribution of electron density that include: ionosondes (Schunk and Nagy 2009), incoherent scatter radars (Kelley 2009) and ionospheric tomography based on the dual-frequency GPS signals (Garcia-Fernandez 2004).

Radio occultation (RO), which is a new remote sensing technique to explore the atmosphere, was first used in NASA's planetary missions to probe the planetary atmosphere (Fjeldbo and Eshleman 1968). In 1975, RO was applied to study the Earth's atmosphere using communications satellites (Feng 2010). Then GPS satellites were considered as signal sources for the proof-of-concept RO missions. The GPS RO concept was successfully demonstrated for the first time by GPS/MET experiment in 1995 (Ware et al. 1996). Since then, much useful occultation data has been collected by many other missions such as the ORSTED; the Stellenbosch University 6 Satellite (SUNSAT); the Challenging Mini-Satellite Payload (CHAMP); Satellite de Aplicaciones Cientificas-C (SAC-C) which have contributed to more than 400 daily occultations (Hajj et al. 2004); the Formosa Satellite-3 and Constellation Observing System for Meteorology, Ionosphere and Climate (FORMOSAT-3/COSMIC); and finally the recent Gravity Recovery and Climate Experiment (GRACE). The main advantages of the RO method are global coverage, high vertical resolution and all-weather capability combined with high accuracy, thereby paving the way for various applications in atmospheric/ionospheric research (e.g., Hajj et al. 2000; Ratnam et al. 2004; Wickert et al. 2004; Wang et al. 2004; Kuo et al. 2005).

The GPS RO technique relies on accurate dual frequency phase measurements by a LEO satellite. An occultation occurs when a GPS global navigation satellite systems (GNSS) satellite rises or sets across the limb with respect to a LEO satellite. The GNSS signal is refracted on its way from the GNSS satellite to the receiver on the LEO satellite while passing the ionosphere. The refraction angle depends on the number of free electrons in the ionosphere. This property is used in the occultation method to determine the ionospheric electron densities (Liou et al. 2007). The most important advantage of RO in comparison with other methods, such as ionosonde stations and ground-based GNSS receivers is its high vertical resolution. Full details of the processing methods to derive the slant total electron content (STEC) between LEO and GPS satellites can be found in Skone (1998), Hajj et al. (2000), Seeber (2003) and Schaer (1999). The inversion methods to convert TEC into the vertical profiles of electron density which contain discrete electron density observations that structurally describe the electron density distribution along the measured points, have been described in COSMIC Data Analysis and Archival Center (CDAAC) (http://www.cosmic.ucar.edu) and Garcia-Fernandez (2004).

Chapman profile function can be applied to describe the vertical structure of the electron density in the ionosphere and it is one of the best-known ionospheric electron density models, which has been successfully used in several applications for the planetary ionosphere modeling, developed by Sidney Chapman in 1931. The original Chapman layer was introduced in the 1960s by Rishbeth and Garriot (1969) for the modeling of the ionospheric electron density for a fixed atmospheric scale height. Reinisch et al. (2007) introduced a modification of the original $\alpha$-Chapman layer with a continuously varying scale height to 
model the F2 topside electron density (The F2 layer is one of the ionospheric layer which is extending from 200 to $1000 \mathrm{~km}$, with an electron density peak at around $300 \mathrm{~km}$ ). Limberger et al. (2013) modeled the maximum electron density $N m F 2$, peak height $h m F 2$ and scale height HF2 of the F2 layer by employing a model approach to the regional applications realized by the combination of the endpoint-interpolating polynomial B splines with an adapted physics-motivated Chapman layer. In this research, the electron density was modeled by applying the multi-layer Chapman profile function for the bottom-side and top-side of the ionosphere and the plasmasphere model. For modeling of the spatio-temporal variations of the maximum electron density of the F2 layer (NmF2) and the corresponding $\mathrm{F} 2$ peak height $(h m F 2)$ in the Chapman function, three-dimensional base functions were defined as a tensor product of the spherical Slepian function for the longitude and latitude and the polynomial B-spline function for time. In this case, the coefficients of the two sets of the Slepian functions are unknown.

\section{Methodology}

In this paper the multi-layer Chapman profile function ( $\alpha$-Chapman function and $\beta$ Chapman function, Chiu 1975; Anderson 1987; Ezquer et al. 1996) and the plasmasphere model proposed by Jakowski (2005) was considered to represent electron density at height $h$ :

$$
\begin{gathered}
N_{e}(h)=\underbrace{N m F 2 \exp \left[0.5\left(1-z-e^{-z}\right)\right]}_{\alpha \text {-Chapmanfunction }}+\underbrace{N m F 2 \exp \left[\left(1-z-e^{-z}\right)\right]}_{\beta-\text { Chapman function }}+\underbrace{N_{P 0} e^{\left(\frac{-h}{H_{p}}\right)}}_{\text {Plasmasphere basis density }} \\
z=\frac{h-h m F 2}{H}
\end{gathered}
$$

The electron density at any point depends on the four unknown parameters in Eq. (1): the F2-layer maximum electron density $N m F 2$, its corresponding height $h m F 2$, the plasmasphere basis density $N_{P 0}$, and the plasmasphere scale height $H_{p}$. In Eq. (1) the bottom-side and topside ionosphere scale height $H$ is assumed to be dependent only on the $h m F 2$ and is given by (Feltens 1998):

$$
H=\frac{h m F 2-50}{3}
$$

where $h m F 2$ is in $\mathrm{km}$. In this research the plasmaspheric parameters are assumed to be known. In accordance with Jakowski (2005) the value for the plasma scale height $H_{p}$ is fixed at $10^{4} \mathrm{~km}$, and the plasmasphere basis density $N_{P 0}$ is set equal to the electron density of the highest topside ionosphere, i.e. electron density at the height of $1000 \mathrm{~km}$. In the presented formulation, the plasmasphere term is simply accounting for a smooth transition of the F2 layer into the plasmasphere (Limberger et al. 2013). With this assumption, the unknown parameters will be restricted to $N m F 2$ and $h m F 2$ which can be modeled in terms of tensor product of the spherical slepian function for the longitude and latitude and the polynomial B-spline function for time. Therefore, the new unknown parameters will be the coefficients of the B-spline and the spherical Slepian functions.

One approach to electron density modeling is based upon the spherical harmonic expansions, which are known as global base-functions. Moreover, their efficient 
application involves the regularly distributed global data. On the other hand, to explain the slight variations in the ionosphere, the increasing of the order and degree of the spherical harmonics is essential. This decreases the computational efficiency, particularly for the real-time applications because of the large size of the observation equations in the computation process. The bias introduced through the data cut-off at the boundaries (the Gibbs phenomenon) is another drawback to the regional modeling of ionosphere using the global support functions (Mautz et al. 2005; Schmidt 2007).

The so-called spatiospectral concentration problem is to determine an orthogonal family of the strictly bandlimited functions optimally concentrated within a closed region of the sphere or to determine an orthogonal family of the strictly spacelimited functions optimally concentrated in the spherical harmonic domain (Simons et al. 2006). The resulting Slepian basis function can be applied efficiently to represent and analyze the regional signals. With the spherical Slepian functions (Wieczorek and Simons 2005; Simons et al. 2006), the tradeoff between spectral and spatial concentrations on the surface of the unit sphere is optimized by constructing a particular linear combination of the spherical harmonics, and the global signals can be decomposed effectively into the regional models which best approximate the field and separate it over the areas of interest, and whose sphericalharmonic spectrum can be studied robustly.

\subsection{Spherical Slepian function}

For each point $r$ on a unit sphere $\Omega$ the real spherical harmonics $Y_{l m}(\mathrm{r})$ of degree $l$ and order $m$ with colatitude $\theta$ and longitude $\lambda$ are defined as:

$$
Y_{l m}(\theta, \lambda)= \begin{cases}\sqrt{2} X_{l|m|}(\theta) \cos m \lambda & \text { if }-l \leq m<0 \\ X_{l 0}(\theta) & \text { if } m=0 \\ \sqrt{2} X_{l m}(\theta) \sin m \lambda & \text { if } 0 \leq m<l\end{cases}
$$

where

$$
X_{l m}(\theta)=(-1)^{m}\left(\frac{2 l+1}{4 \pi}\right)^{1 / 2}\left[\frac{(l-m) !}{(l+m) !}\right]^{1 / 2} P_{l m}(\cos \theta)
$$

and $P_{l m}(\mu)$ is an associated Legendre polynomial. These spherical functions form a set of the orthonormal basis for square-integrable and real-valued functions on the unit sphere. In other words, every square-integrable function can be described as a unique linear combination of the spherical harmonics expansion (Wieczorek 2007):

$$
f(r)=\sum_{l=0}^{\infty} \sum_{m=-l}^{l} f_{l m} Y_{l m}(r)
$$

where $f_{l m}$ is the expansion coefficient obtained as:

$$
f_{l m}=\frac{1}{4 \pi} \int_{\Omega} f(r) Y_{l m}(r) \mathrm{d} \Omega .
$$

In order to localize the spherical harmonic functions into a region of interest $R$ (target region), the optimization of a local energy criterion (Eq. 10) can be utilized. This will give a new set of functions in the sense of Slepian (1983). They are band-limited to a maximum spherical harmonic degree $L$ and, at the same time, are spatially concentrated inside a 
target region. In other words, the Slepian basis set is merely a unitary linear transformation of the spherical-harmonic basis, but it is the spatial region of interest, built into their construction via quadratic maximization, that leads to their efficiency for the modeling of the regional signals (Beggan et al. 2013). The spherical Slepian function can be presented as a band-limited spherical harmonic expansion:

$$
g(r)=\sum_{l=0}^{L} \sum_{l=-m}^{m} g_{l m} Y_{l m}(r)
$$

with:

$$
g_{l m}=\int_{\Omega} g(r) Y_{l m} d \Omega .
$$

Which is obtained by maximizing the energy concentration:

$$
\lambda=\max \frac{\int_{R} G^{2}(\Omega) d \Omega}{\int_{\Omega_{G}} G^{2}(\Omega) d \Omega}
$$

where $0 \leq \lambda \leq 1$. The maximization of this concentration criterion can be achieved in the spectral domain by solving an eigenvalue problem (Simons et al. 2006):

$$
D g=\lambda g
$$

where the elements of $(L+1)^{2} \times(L+1)^{2}$ localizing kernel $D$ :

$$
D=\left(\begin{array}{ccc}
D_{00,00} & \cdots & D_{00, \mathrm{LL}} \\
\cdot & & \cdot \\
\cdot & & \cdot \\
D_{\mathrm{LL}, 00} & \cdots & D_{L L, \mathrm{LL}}
\end{array}\right)
$$

are given by

$$
D_{l m, l^{\prime} m^{\prime}}=\int_{R} Y_{l m}(r) Y_{l^{\prime} m^{\prime}}(r) d \Omega .
$$

And $g$ is the $(L+1)^{2}$ dimensional vector that represents a Slepian eigenfunction expressed in the spherical harmonics, i.e.:

$$
g=\left(g_{00} \ldots g_{l m} \ldots g_{L L}\right)^{T}
$$

when the signal $g(r)$ is local, it can be approximated using the Slepian expansion truncated at the Shannon number $N$ (Percival and Walden 1993):

$$
N=\sum_{n=1}^{(L+1)^{2}} \lambda_{n}=(L+1)^{2} \frac{A}{4 \pi}
$$

where $A$ is the area of the region as a fraction of the full sphere. The data can be approximated, yet with very good reconstruction properties within the region by (Simons 2010): 


$$
d(\hat{r}) \approx \sum_{n=1}^{N} d_{n} g_{n}(\hat{r})
$$

where $g_{n}(\hat{r})$ and $d_{n}$ are the spherical Slepian function and unknown coefficient, respectively. To model the unknown parameters by means of the Slepian function, the following equations can be written (Sharifi and Farzaneh 2013):

$$
\begin{aligned}
& N_{m}(\beta, s)=\sum_{l=0}^{L} \sum_{m=-l}^{l} \omega_{l m} Y_{l m}(\varphi, \lambda)=\sum_{n=1}^{(L+1)^{2}} \omega_{n} g_{n}(\varphi, \lambda) \approx \sum_{n=1}^{N} \omega_{n} g_{n}(\varphi, \lambda) \\
& h_{m}(\beta, s)=\sum_{l=0}^{L} \sum_{m=-l}^{l} \omega_{l m}^{\prime} Y_{l m}(\varphi, \lambda)=\sum_{n=1}^{(L+1)^{2}} \omega_{n}^{\prime} g_{n}(\varphi, \lambda) \approx \sum_{n=1}^{N} \omega_{n}^{\prime} g_{n}(\varphi, \lambda)
\end{aligned}
$$

where $Y_{l m}(\varphi, \lambda), g_{n}(\varphi, \lambda)$ and $\omega\left(\omega^{\prime}\right)$ are the spherical harmonic, Slepian function and the unknown coefficient. The Eqs. (17) and (18) can be written as:

$$
\begin{aligned}
& N_{m_{i}}=N_{m}\left(\varphi_{i}, \lambda_{i}\right)=a_{i}^{T} x \\
& h_{m_{i}}=h_{m}\left(\varphi_{i}, \lambda_{i}\right)=a_{i}^{T} x^{\prime}
\end{aligned}
$$

In which $a_{i}=a\left(\varphi_{i}, \lambda_{i}\right)=g_{n}\left(\varphi_{i}, \lambda_{i}\right)$ is the design matrix containing the Slepian functions, and $\boldsymbol{x}=\left[\omega_{1}, \omega, \ldots, \omega_{(L+1)^{2}}\right]^{T}, \boldsymbol{x} \prime\left[\omega_{1}^{\prime}, \omega_{2}^{\prime}, \ldots \omega_{(L+1)^{2}}^{\prime}\right]$ are the unknown coefficients.

For the time dependent modeling, the unknown parameters have to be represented as a function of longitude, latitude and time by means of the Slepian function. Now for the sake of brevity the equations are obtained only for $N_{m}$ and the same procedure is repeated for $h_{m}$. Equation (17) is rewritten and obtained as follows:

$$
N_{m}\left(\varphi_{i}, \lambda_{i}, t_{q}\right)=N_{m_{i}}\left(t_{q}\right)=a_{i}^{T} x_{q}
$$

For $q=1 \ldots Q$ with $x\left(t_{q}\right)=x_{q}$. If Eq. (21) is written for $\mathrm{i}=1 \ldots 1$, then:

$$
N_{m_{q}}+R_{q}=A X_{q}
$$

where $R_{\mathrm{q}}$ is the measurement error and $A$ is the coefficient matrix defined as $\mathbf{A}=$ $\left[a_{1}, a_{2}, \ldots, a_{l}\right]^{T}$. Since the time dependency is propagated into the series of coefficients $\omega_{n}\left(t_{q}\right)$ (the components of the vector $X_{\mathrm{q}}$ ), the polynomial B-Spline expansion has been introduced as follows:

$$
T(t)=\sum_{k=0}^{k_{j}-1} C_{k}^{j} \Phi_{k}^{j}(t)
$$

where $\Phi_{k}^{j}(t)$ is the one-dimensional normalized quadratic B-Spline scaling function of the level j and shift k (Stollnitz et al. 1995a, 1995b; Lyche and Schumaker 2000) and $C_{j}^{\prime k}$ is the unknown series coefficients (Schmidt 2007). By defining $T\left(t_{q}\right)=\omega_{n}\left(t_{q}\right)$ and $C_{j}^{\prime k}=\omega_{n, k}^{j}$ the following equation is obtained:

$$
\omega_{n}\left(t_{q}\right)=\sum_{k=0}^{k_{j}-1} \omega_{n, k}^{j} \Phi_{k}^{j}\left(t_{q}\right)
$$

where $\omega_{n}\left(t_{q}\right)$ is the time-dependent unknown coefficient in Eq. (17). By writing Eq. (22) in the matrix form, the equation below is obtained: 


$$
\omega_{q}=\Omega U_{q}
$$

where the $(L+1)^{2} \times K_{j}$ matrix $\boldsymbol{\Omega}$ is defined as:

$$
\Omega=\left[\begin{array}{cccc}
\omega_{1,0}^{j} & \omega_{1,1}^{j} & \ldots & \omega_{1, k_{j}-1}^{j} \\
\vdots & & & \vdots \\
\omega_{(L+1)^{2}, 0}^{j} & \omega_{(L+1)^{2}, 1}^{j} & \ldots & \omega_{(L+1)^{2}, k_{j}-1}^{j}
\end{array}\right] .
$$

The $K_{j} \times 1$ vector $\boldsymbol{U}_{q}=\boldsymbol{U}\left(t_{q}\right)$ is:

$$
U_{q}=\left[\Phi_{0}^{j}\left(t_{q}\right), \Phi_{1}^{j}\left(t_{q}\right), \ldots, \Phi_{k_{j}-1}^{j}\left(t_{q}\right)\right]^{T}
$$

Since the variable $t$ in the scaling function $\Phi_{j}^{k}(t)$ takes values at the unit interval $[0,1]$, the variable $t$ is transformed into this interval using:

$$
t=\frac{t^{\prime}-t_{\min }^{\prime}}{t_{\max }^{\prime}-t_{\min }^{\prime}}
$$

where $t_{\min }^{\prime}$ and $t_{\max }^{\prime}$ are the starting and ending epochs of the modeling interval. By inserting Eq. (25) into Eq. (22), the following equation is obtained:

$$
Y+R=A \Omega U
$$

In which $\mathrm{Y}=\left[y_{1}, y_{2}, \ldots y_{\mathrm{Q}}\right]$ is the $l \times Q$ observation matrix, $\boldsymbol{R}=\left[r_{1}, r_{2}, \ldots, r_{\mathrm{Q}}\right]$ is the observation error matrix, and $\mathrm{U}=\left[u_{1}, u_{2}, \ldots, u_{Q}\right]$ is the $K_{j} \times Q$ B-Spline scaling function matrix. For the determination of the Slepian coefficients a least-squares approach is established. The observation equation given by Eq. (1) is nonlinear. To facilitate the solution, a first-order Taylor series approximation can be used to create a set of linear equations:

$$
l_{i}+r_{i}=f_{i}\left(x_{0}\right)+\left.\frac{\partial f_{i}}{\partial x}\right|_{x=x_{0}} \delta x=B \delta x=K+r
$$

In this case the correction $\delta x$ to the approximate value $x_{0}$ is obtained, where $x_{0}$ denotes the vector of initial value of the variable.

In Eq. (30) $r$ indicates observation noise, $l$ is the $n \times 1$ vector that contains the observation, and matrix $B$ contains partial derivatives of the observation equation with respect to the unknown parameters. The partial derivatives in matrix $B$ are evaluated for each of the series of $n$ observation using the latest estimates $x_{0}$ of the parameters $x$. Table 1 indicate the linearization of the observation equation, and $K$ is the reduced observation vector $l$, which contains the differences between observed and computed initial electron densities. Since the data distribution of electron density profiles is rather sparse, some constraints should be applied or the data gaps need to be bridged by priori information from a background model to avoid obtaining unrealistic large residuals. In this study the initial values $x_{0}$ for the series coefficients have been derived from IRI-2012 model (bilitza et al. 2011) and the data source for the prior information has been obtained from South Africa's bottomside ionosphere model (McKinnell and Poole 2004). 
Table 1 Linearization of the observation equation

\begin{tabular}{|c|c|}
\hline $\begin{array}{l}\text { The partial derivative with respect to } \omega_{n, k}^{j} \\
\qquad \frac{\partial N}{\partial \omega_{n, k}^{j}}=\frac{\partial N}{\partial N_{m}} \frac{\partial N_{m}}{\partial \omega_{n}} \frac{\partial \omega_{n}}{\partial \omega_{n, k}^{j}}\end{array}$ & $\begin{array}{l}\text { The partial derivative with respect to } \omega_{n, k}^{j j} \\
\frac{\partial N}{\partial \omega_{n, k}^{j j}}=\frac{\partial N}{\partial h_{m}} \frac{\partial h_{m}}{\partial \omega_{n}^{\prime}} \frac{\partial \omega_{n}^{\prime}}{\partial \omega_{n, k}^{j}}\end{array}$ \\
\hline $\begin{aligned} \frac{\partial N}{\partial N_{m}}= & \exp \left[\alpha\left(1-z-e^{-z}\right)\right]_{\text {bottomside }} \\
& +\exp \left[\beta\left(1-z-e^{-z}\right)\right]_{\text {topside }}\end{aligned}$ & $\begin{aligned} \frac{\partial N}{\partial h_{m}}= & -3 N_{m}\left(e^{-z}-1\right)\left(\frac{h-50}{(h m F 2-50)^{2}}\right) \\
& \times\left(\alpha \exp \left[\alpha\left(1-z-e^{-z}\right)\right]_{\text {bottomside }}\right. \\
& \left.+\beta \exp \left[\beta\left(1-z-e^{-z}\right)\right]_{\text {topside }}\right)\end{aligned}$ \\
\hline$\frac{\partial N_{m}}{\partial \omega_{n}}=g_{n}(\varphi, \lambda)$ & $\frac{\partial h_{m}}{\partial \omega_{n}^{\prime}}=g_{n}^{\prime}(\varphi, \lambda)$ \\
\hline$\frac{\partial \omega_{n}}{\partial \omega_{n, k}^{j}}=\Phi_{k}^{j}\left(t_{q}\right)$ & $\frac{\partial \omega_{n}^{\prime}}{\partial \omega_{n, k}^{j}}=\Phi \prime_{k}^{j}\left(t_{q}\right)$ \\
\hline
\end{tabular}

\section{Results and discussion}

The FORMOSAT-3/COSMIC constellation of 6 satellites was launched on 15 April, 2006. Their initial orbits were at an altitude of $500 \mathrm{~km}$, but they were gradually raised to an altitude of $800 \mathrm{~km}$. A separation between the satellites is $30^{\circ}$, with an orbital period of 100 min (Liou et al. 2007). More than 3 million ionospheric profiles have hitherto been provided by the COSMIC working group. In this study, the second level data provided by CDACC - "ionprf" files containing information about ionospheric electron densities from http://www.cosmic.ucar.edu with a reported accuracy of $0.1-1 \times 10^{11}$ ("COSMIC Program Office Website" 2013) was used. South Africa, located between the geographical latitudes of $-12^{\circ}$ and $-37^{\circ}$, and geographical longitudes of $10^{\circ}$ and $40^{\circ}$ East was selected as our study region. To demonstrate the performance of the proposed technique, the model is validated under different ionospheric conditions, different locations (in different latitude and longitude) and different season. By this evaluation we consider (a) Solar cycle, (b) Seasonal, (c) Diurnal and (d) Latitude dependent variations. To conduct the analysis, certain times were picked. This time interval is selected intentionally in order to model the electron density which is strongly influenced by mentioned variations. Figures 1 and 2 show the footprints of $\mathrm{F} / \mathrm{C}$ occultation measurements (blue crosses) and geomagnetic conditions for 20-22 August 2008 (Solar minimum ionospheric condition), 28-30 June 2009 (Solar moderate ionospheric condition), 21-23 November 2009 (Solar minimum ionospheric condition) and 05-07 August 2011 (Solar maximum ionospheric condition), respectively. As a result of the spherical symmetry assumption in the Abel transform which is used in the retrieval of the COSMIC ionospheric profiles and the existence of the horizontal gradients of the electron density, the profiles are not realistic at low altitudes (Hajj et al. 2000). Furthermore, from the investigation of the data, it was found that some measurements at high altitudes (more than $500 \mathrm{~km}$ ) are also unreliable. Since most electron densities are concentrated between 150 and $500 \mathrm{~km}$ altitudes, the electron densities for the low altitudes $(<150 \mathrm{~km})$ and high altitudes $(>500 \mathrm{~km})$ are ignored. Before proceeding to the electron density modeling, it is necessary to perform some quality control tests on the individual ionospheric electron density profiles, and to remove bad profiles. For this purpose, each profile was fitted to a two-layer Chapman function described in Lei et al. (2007) using the least-squares method. (i.e. A least-squares fitting of the RO electron density profile at the F2 layer to a two-layer Chapman function is performed). This brings 
(a)

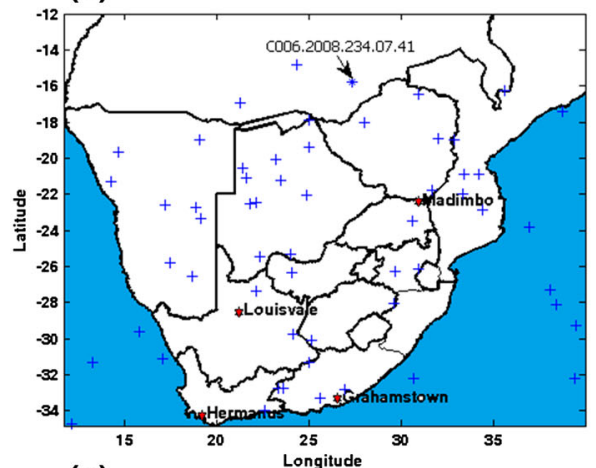

(c)



(b)

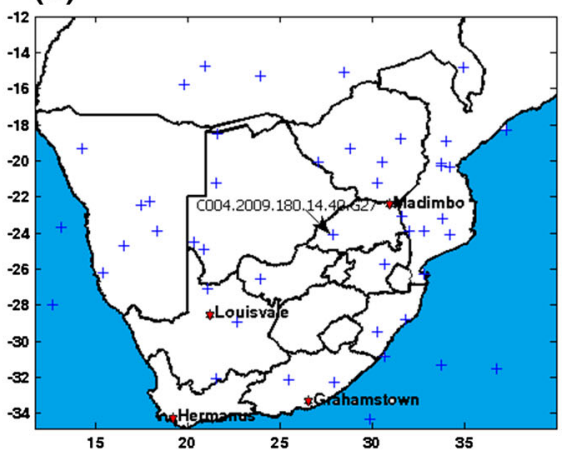

(d)

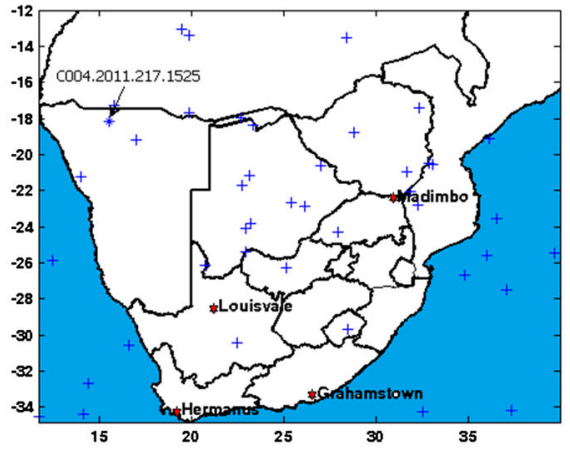

Fig. 1 Footprints of F/C occultation measurements for a 20-22 August 2008, b 28-30 June 2009, c 21-23 November 2009 and d 05-07 August 2011

in the best match with $\mathrm{RO}$ electron density profiles at F2 region. Furthermore, in order to quantitatively assess the effect of ionospheric plasma irregularities on the height variation of the electron density, Yang et al. (2009), define mean deviation of the electron density profile as follows:

$$
\text { Mean deviation }=\sum_{i} \frac{\left|n_{i}-\bar{n}_{i}\right|}{N \bar{n}_{i}}
$$

where $N, n_{i}$ and $\bar{n}_{i}$ are the total data points in a profile, the measured electron densities at the i-th height and the mean electron densities at the i-th height by taking 9-point running average of the measured electron density profile, respectively. In this study the profiles in which the obtained mean deviations were greater than 1.5 , considered as unsuitable electron density profiles.

Figure 3 illustrates the two samples related to a suitable and unsuitable electron density profiles. In this research the accepted electron density profiles at six four-hourly time frames on 21 August 2008, 29 June 2009, 21 November 2009 and 05 August 2011, were used to model the electron density profiles.

Accuracy of the estimated parameters has been assessed in several aspects. First the comparison between the Model- $h m F 2 / N m F 2$ values, the IRI-2012- $h m F 2 / N m F 2$ values, and the ionosonde- $h m F 2 / N m F 2$ values from the Grahamstown $\left(\varphi=-33.3^{\circ}, \lambda=26.5^{\circ}\right)$, Louisvale $\left(\varphi=-28.5^{\circ}, \lambda=21.2^{\circ}\right)$, Madimbo $\left(\varphi=-22.4^{\circ}, \lambda=30.9^{\circ}\right)$ and Hermanus 
(a)

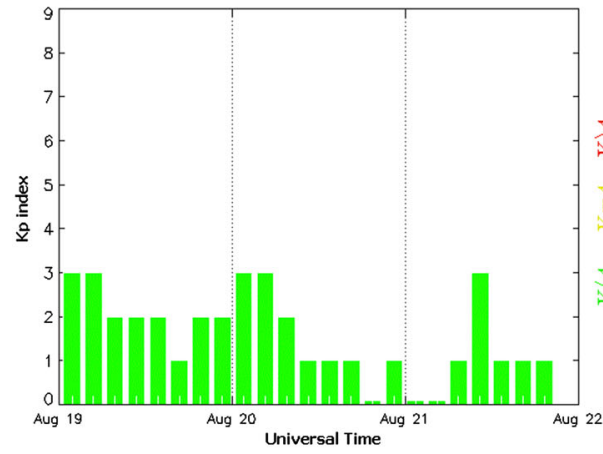

(c)



(b)

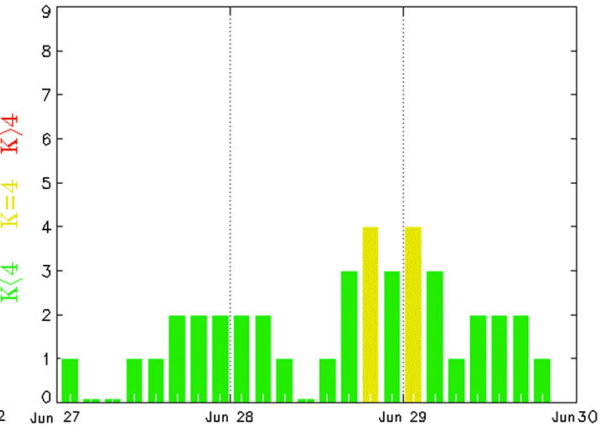

(d)

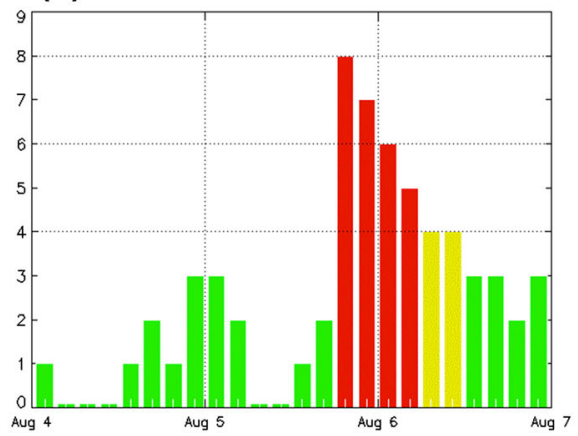

Fig. 2 The estimated planetary K index for a 20-22 August 2008, b 28-30 June 2009, c 21-23 November 2009 and d 05-07 August 2011 (http://www.spaceweatherlive.com)


Electron Density (el/cm3)

Fig. 3 Left the failed electron density profile; Right the accepted electron density profile observed by FORMOSAT-3/COSMIC in quality control tests

$\left(\varphi=-34.2^{\circ}, \lambda=19.2^{\circ}\right)$ ionosonde stations have been made. Tables 2 and 3 illustrate the average of the absolute percentage deviations (AAPD) of the Model- $h m F 2 / N m F 2$ values and the IRI-2012- $h m F 2 / N m F 2$ values from the ionosonde- $h m F 2 / N m F 2$ values, using the following equation: 
Table 2 Deviation of the Model values and the IRI-2012 values from ionosonde values for $h m F 2$, day 218, 2011

\begin{tabular}{|c|c|c|c|c|}
\hline \multirow{2}{*}{$\begin{array}{l}\text { Ionosonde station } \\
\text { Evaluated parameter }\end{array}$} & \multicolumn{2}{|c|}{$\begin{array}{l}\text { Average of the absolute percentage } \\
\text { deviations of the model values } \\
\text { deviations from the Ionosonde } \\
\text { values }(\%)\end{array}$} & \multicolumn{2}{|c|}{$\begin{array}{l}\text { Average of the absolute percentage } \\
\text { deviations of the IRI-2012 values } \\
\text { deviations from the Ionosonde values }(\%)\end{array}$} \\
\hline & $h m F 2$ & $N m F 2$ & $h m F 2$ & $N m F 2$ \\
\hline Grahamstown & 2.012 & 1.825 & 5.715 & 2.291 \\
\hline Louisvale & 1.025 & 1.536 & 2.485 & 3.047 \\
\hline Madimbo & 1.086 & 1.023 & 2.140 & 4.791 \\
\hline Hermanus & 1.945 & 2.012 & 2.758 & 3.041 \\
\hline
\end{tabular}

Table 3 Deviation of the model values and the IRI-2012 values from ionosonde values for $h m F 2$, day 234, 2008

\begin{tabular}{|c|c|c|c|c|}
\hline \multirow[t]{2}{*}{ Ionosonde station } & \multicolumn{2}{|c|}{$\begin{array}{l}\text { Average of the absolute percentage } \\
\text { deviations of the model values deviations } \\
\text { from the Ionosonde values }(\%)\end{array}$} & \multicolumn{2}{|c|}{$\begin{array}{l}\text { Average of the absolute percentage } \\
\text { deviations of the IRI- } 2012 \text { values } \\
\text { deviations from the Ionosonde values }(\%)\end{array}$} \\
\hline & $h m F 2$ & $N m F 2$ & $h m F 2$ & $N m F 2$ \\
\hline Grahamstown & 7.773 & 4.168 & 13.795 & 9.127 \\
\hline Hermanus & 7.585 & 3.843 & 12.312 & 6.027 \\
\hline
\end{tabular}

$$
A A P D=\frac{\sum_{i=1}^{n}\left(100 \times\left|\frac{x_{i}-x_{i}^{\prime}}{x_{i}}\right|\right)}{n}
$$

where $x_{i}$ represents the ionosonde- $h m F 2 / N m F 2$ values, the $x^{\prime}$ represent the Model- $h m F 2 /$ $N m F 2$ values or the IRI-2012- $h m F 2 / N m F 2$ values, and $n$ represents the sample size. Considering the 15 min time interval related to the ionosonde stations observations, the sample size is 96. It is noteworthy that in Table 3 due to lack of the Madimbo and Louisvale observations, the validation has been performed at the remaining stations. The results show a better correlation between the model values and the ionosonde values than the correlation between the IRI-2012 and the ionosonde.

Second, the model estimated by RO observations was evaluated by the four specified profiles in Fig. 1, which did not participate in modeling. Many authors have investigated the accuracy of electron density profile derived from RO measurements: Yue et al. (2013) reported an accuracy of $0.5 \times 10^{11} \mathrm{el} / \mathrm{m} 3$, Jakowski et al., (2005) reported a systematic positive bias in the order of less than $0.8 \times 10^{11} \mathrm{el} / \mathrm{m} 3$ and a standard deviation of $1.3 \times 10^{11} \mathrm{el} / \mathrm{m} 3$. In this research, the accuracy of $1 \times 10^{11} \mathrm{el} / \mathrm{m} 3$ reported by COSMIC Program Office Website is used as a reference of the accuracy measure of the RO retrieved electron density profiles.

Figure 4 shows the comparison between four RO events at the time frame between (a) 06:00-10:00 UT on 21 August 2008, (b) 12:00-16:00 on 05 August 2011, (c) 12:00-16:00 UT on 29 June 2009 and (d) 12:00-16:00 UT on 21 November 2009, for observed and estimated electron density profiles, each subplot shows the difference between two derived profiles. The red dotted line represent the accuracy range 
(a)



(c)

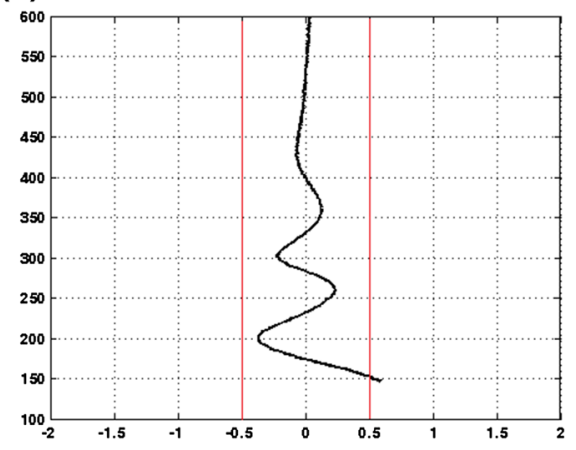

(b)



(d)



Fig. 4 The comparison between estimated and observed electron density for a Year 2008 Day Number 234, b Year 2011 Day Number 217, c Year 2009 Day Number 180 and d Year 2009 Day Number 325

$\left( \pm 1 \times 10^{11} \mathrm{el} / \mathrm{m} 3\right)$ of the RO electron density profile, the figure depicts the residuals are within the range of RO accuracy.

\section{Conclusion}

Precise ionosphere modeling is crucial and remains as a challenge for GPS positioning and navigation, as well as many other Earth observation systems. The GPS RO techniques (RO) are effective tools to study the layered ionospheric structures. In this study, the spherical Slepian function, which has not, thus far, been applied to the local modeling of the electron density in the literature, was performed for the real sets of data obtained from the FORMOSAT-3/COSMIC satellites across the South Africa. In this research, in order to model the electron density in longitude, latitude and time, the three-dimensional base functions were defined as a tensor product of the spherical Slepian function for the longitude and latitude and the polynomial B-spline function for time. For height dependency, the Chapman profile function was used to express the height variations of the electron density. To model the parameters of the Chapman profile function locally, the two sets of spherical Slepian functions were implemented. After obtaining the unknown coefficients of the spherical Slepian functions, the F2 maximum electron density and its corresponding height were obtained. The estimated F2-peak values are compared with the IRI model 
values, and those obtained from ionosonde at Grahamstown, Louisvale, Madimbo and Hermanus stations. In addition the estimated model was validated by some profiles, which did not participate in modeling. Validations show the ability of this model approach to yield reliable results.

Acknowledgments The authors would like to acknowledge (1) the NOAA National Geophysical Data Center/NGDC for the Ionosondes data (http://spidr.ngdc.noaa.gov/spidr/); (2) the NPSO (Taiwan's National Space Organization) and UCAR (University Center for Atmospheric Research) for access the COSMIC RO data (http://cdaac-www.cosmic.ucar.edu/cdaac/products.html).

\section{References}

Anderson DN, Mendillo, Hertniter B (1987) A semiempirical low latitude ionospheric model. Radio Sci 22:292-306

Beggan C, Saarimaki J, Whaler K, Simons F (2013) Spectral and spatial decomposition of lithospheric magnetic field models using spherical Slepian functions. Geophys J Int 193:136-148

Bilitza D, McKinnell LA, Reinisch B, Fuller-Rowell T (2011) The international reference ionosphere (iri) today and in the future. J Geodesy 85:909-920

Chiu Y (1975) An improved phenomenological model of ionospheric density. J Atmos Terr Phys 37:1563-1570

Ezquer R, De Gonzalez M, Heredia T (1996) Electron density profile modeling. Annali Di Geofisica, XXXIX, pp 539-542

Feltens J (1998) Chapman profile approach for 3-D global TEC representation. Paper presented at 1998 IGS Analysis Centers Workshop, Eur. Space Op. Cent., Darmstadt

Feng M (2010) Detection of high-latitude ionospheric irregularities from GPS radio occultation. MSc dissertation, University of Calgary

Fjeldbo G, Eshleman VR (1968) The atmosphere of Mars analyzed by integral inversion of the Mariner IV occultation data. Planet Space Sci 16:1035-1059

Garcia-Fernandez M (2004) Contributions to the 3D ionospheric sounding with GPS data. PhD dissertation, Universitat Politecnica de Catalunya (UPC)

Hajj GA, Lee LC, Pi X, Romans LJ, Schreiner WS, Straus PR, Wang C (2000) COSMIC GPS ionospheric sensing and space weather. Terr Atmos Ocean Sci 11(1):235-272

Hajj GA, Ao CO et al (2004) CHAMP and SACC atmospheric occultation results and intercomparisons. J Geophys Res 109:109. doi:10.1029/2003JD003909

Jakowski N (2005) Ionospheric GPS radio occultation measurements on board CHAMP. GPS Solut 9:88-95

Jakowski N, Tsybulyal K, Radicella SM, Cueto M, Herraiz M (2005) Comparison of electron density profiles from CHAMP data with NeQuick model. In: Reigber PDDC, Lühr PDH, Schwintzer DP, Wickert DJ (eds) Earth observation with CHAMP. Springer, Berlin, pp 483-488

Kelley MC (2009) The Earth's ionosphere: plasma physics and electrodynamics, 2nd edn. Academic Press, San Diego

Kuo YH, Schreiner WS, Wang J, Rossiter DL, Zhang Y (2005) Comparison of GPS radio occultation soundings with radiosondes. Geophys Res Lett 32:L05817. doi:10.1029/2004GL021443

Lei JS, Syndergaard AG et al (2007) Comparison of COSMIC ionospheric measurements with ground-based observations and model predictions: preliminary results". J Geophys Res 112:A07308

Limberger M, Liang W, Schmidt M, Dettmering D, Hugentobler U (2013) Regional representation of F2 Chapman parameters based on electron density profiles. J Ann Geophys 31:2215-2227

Liou YA, Pavelyev AG, Liu SF, Pavelyev AA, Yen N, Huang CY, Fong CJ (2007) FORMOSAT-3/ COSMIC GPS radio occultation mission: preliminary results. IEEE Trans Geosci Remote Sens 45(11):3813-3826

Lyche T, Schumaker LL (2000) A multiresolution tensor spline method for fitting functions on the sphere. SIAM J Sci Comput 22(2):724-746

Mautz R, Ping J, Heki K, Schaffrin B, Shum CK, Potts L (2005) Efficient spatial and temporal representations of global ionosphere maps over Japan using B- spline wavelets. J Geodesy 78:660-667

McKinnell L-A, Poole AW (2004) Neural network-based ionospheric modelling over the South African region. S Afr J Sci 100:519-523

Percival DB, Walden AT (1993) Spectral analysis for physical applications, multitaper and conventional univariate techniques. Cambridge University Press, New York 
Ratnam MV, Tsuda T, Jacobi C, Aoyama Y (2004) Enhancement of gravity wave activity observed during a major southern hemisphere stratospheric warming by CHAMP/GPS measurements. Geophys Res Lett 31:L16101

Reinisch BW, Nsumeia P, Huanga X, Bilitza DK (2007) Modeling the F2 topside and plasmasphere for IRI using IMAGE/RPI and ISIS data. Adv Space Res 39:731-738

Rishbeth H, Garriot OK (1969) Introduction to ionospheric physics. Academic Press, New York

Schaer S (1999) Mapping and predicting the Earth's ionosphere using the global positioning system. PhD dissertation, University of Berne

Schmidt M (2007) Wavelet modeling in support of IRI. Adv Space Res 39:932-940

Schunk RW, Nagy AF (2009) In: Houghton JT, Rycroft MJ, Dessler AJ (eds) Ionospheres: physics, plasma physics, and chemistry (2nd edn). Cambridge University Press, New York

Seeber G (2003) Satellite geodesy: foundations, methods, and applications. Walter de Gruyter, Berlin

Sharifi MA, Farzaneh S (2013) The spatio-spectral localization approach to modelling VTEC over the western part of the USA using GPS observations. Adv Space Res 54(6):908-916

Simons FJ (2010) Slepian functions and their use in signal estimation and spectral analysis. In: FreedenW, Nashed MZ, Sonar T (eds) Handbook of geomathematics, pp 891-923. Springer, Berlin

Simons FJ, Dahlen FA, Wieczorek MA (2006) Spatiospectral Concentration on a Sphere. Soc Ind Appl Math Rev 48:504-536

Skone S (1998) Wide area ionosphere grid modelling in the auroral region. PhD dissertation, University of Calgary

Slepian D (1983) Some comments on Fourier-analysis. Uncertainty and modeling. SIAM Rev 25(3):379-393

Stollnitz EJ, DeRose TD, Salesin DH (1995a) Wavelets for computer graphics: a primer. Part I. IEEE Comput Graphics Appl 15(3):76-84

Stollnitz EJ, DeRose TD, Salesin DH (1995b) Wavelets for computer graphics: a primer. Part II. IEEE Comput Graphics Appl 15(4):75-85

Wang DY, Stiller GP et al (2004) Cross-validation of MIPAS/ENVISAT and GPSRO/CHAMP temperature profiles. J Geophys Res 109:D19311

Ware RD, Exner M et al (1996) GPS sounding of the atmosphere from low Earth orbit—preliminary results. Bull Am Met Soc 77:19-40

Wickert JA, Pavelyev G, Liou YA, Schmidt T, Reigber C, Pavelyev AA, Igarashi K, Matyugov S (2004) Amplitude variations in GPS signals as a possible indicator of ionospheric structures. Geophys Res Lett 31:L24801

Wieczorek MA (2007) Gravity and topography of the terrestrial planets. Treatise Geophys 10:165-206

Wieczorek MA, Simons FJ (2005) Localized spectral analysis on the sphere. Geophys J Int 162:655-675

Yang KF, Chu YH, Su CL, Ko HT, Wang CK (2009) An examination of FORMOSAT-3/COSMIC ionospheric electron density profile: data quality criteria and comparisons with the IRI model. Terr Atmos Ocean Sci 20:193-206

Yue X, Schreiner WS, Kuo Y-H, Wu Q, Deng Y, Wang W (2013) GNSS radio occultation (RO) derived electron density quality in high latitude and polar region: NCAR-TIEGCM simulation and real data evaluation. J Atmos Solar-Terr Phys 98:39-49 